الأحمدي : تحديد الثروط المثلى لإزالة القلوريد من مياه الشرب باستخدام طريقة نالكوندا (التخثير والترسيب)

$$
\begin{aligned}
& \text { تحديد الشروط المثلي لإز الة القلوريد من مياه الثرب باستخدام طريقة } \\
& \text { زينب علي زناد } \\
& \text { الكلية التقتية كركوتي }
\end{aligned}
$$

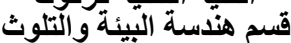

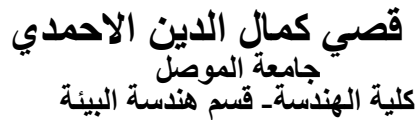

$$
\text { الملخص }
$$

تعد طريقة نالكوندا من الطرق الفعالة في ازالة الفلوريد وخصوصا لتراكيز الفلوريد الاولية القليلة والمتوسطة

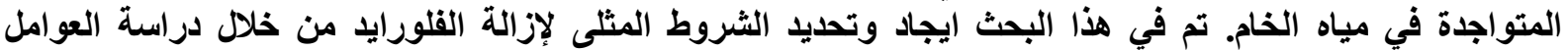

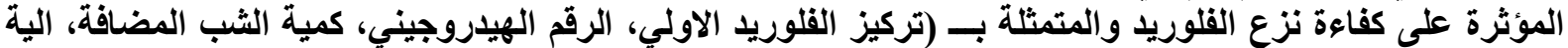

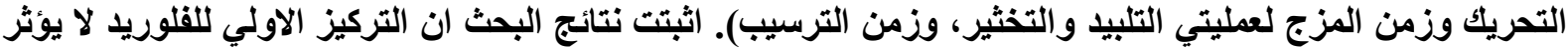

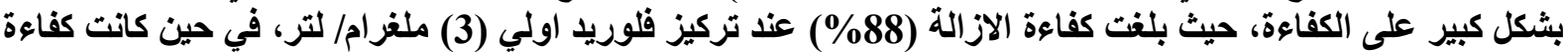

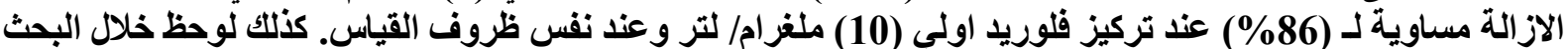

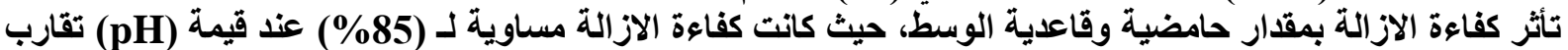

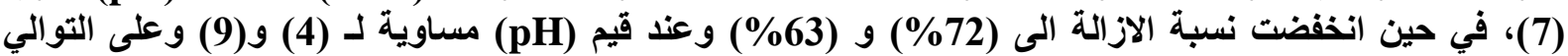

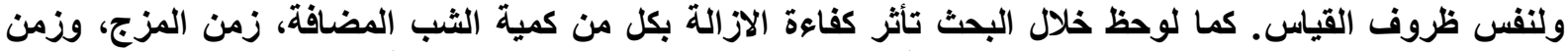

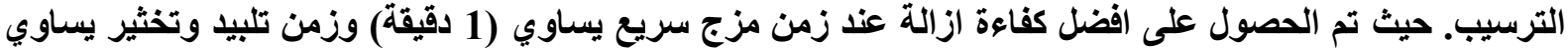

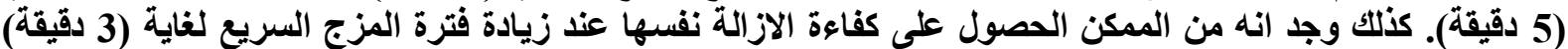

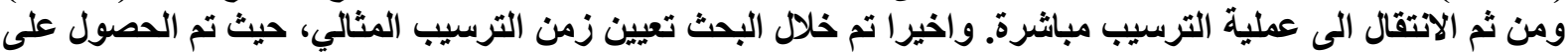

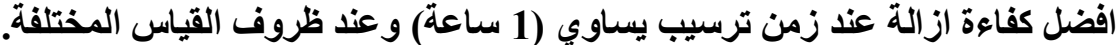

\title{
The Optimum Conditions For Fluoride Removal From Drinking Water Using Nalgonda Method (Flocculation And Sedimentation)
}

\author{
Dr. Kossay K. Al-Ahmady
}

University of Mosul

College of Engineering, Environmental Eng. Department

\author{
Zainab A. Znad
}

College of Technology, Kirkuk Environment and Pollution Eng. Department

\section{Abstract}

The Nalgonda method is one of the effective methods for fluoride removal, especially with low and medium initial fluoride concentrations. In this research, the ideal conditions were found through studying the factors that affect the fluoride removal efficiency (initial fluoride concentration, $\mathrm{pH}$, alum dose, mixing time for flocculation and coagulation processes, and the sedimentation time). The research results proved that initial fluoride concentration does not significantly effect the efficiency. As the removal efficiency amounted to $(88 \%)$ for the initial fluoride concentration of $(3) \mathrm{mg} / \mathrm{L}$. While the removal efficiency was equal to $(\mathbf{8 6} \%)$ for an the initial fluoride concentration of (10) $\mathrm{mg} / \mathrm{L}$. pH value was proved to be effective, as the removal efficiency was equal to $(85 \%)$ when the $(\mathrm{pH})$ value is equal to $(7)$, while the removal decreased to $(72 \%)$ and $(63 \%)$ when the (pH) values were equal to (4) and (9) respectively. Alum dose, time of mixing, and the time of sedimentation were also affecting on the removal efficiency. Best removal efficiency obtained at the times of mixing and flocculating equal to $(1 \mathrm{~min})$ and (5 min) respectively. Also it was found that it's possible to get the same removal efficiency when increasing the period of rapid mixing to $(3 \mathrm{~min})$ and then directly move to the sedimentation process. Finally, the ideal sedimentation time has been set, best removal efficiency has been achieved a sedimentation time equal to (1 hour) and at the different measurement conditions.

Keywords: Flouride removal, Drinking water, Nalgonda method, Flocculation, Sedimentation 


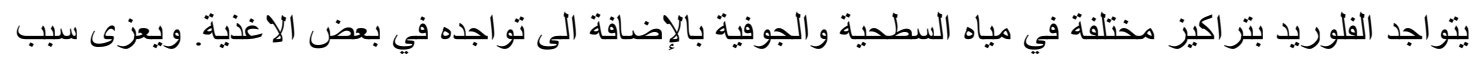

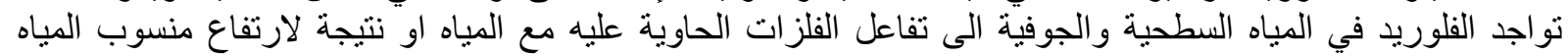

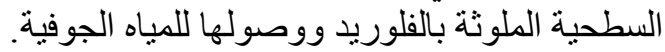

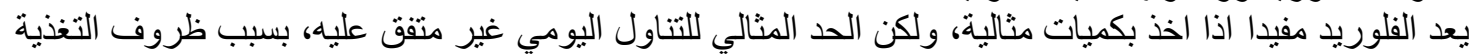

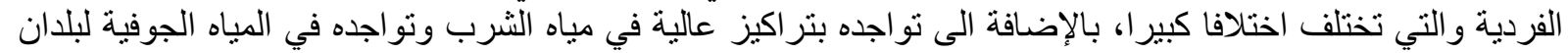

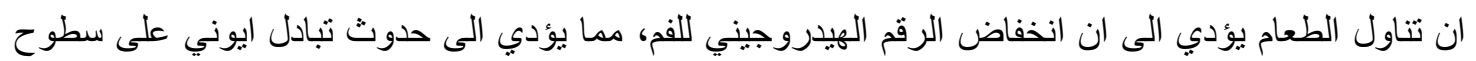

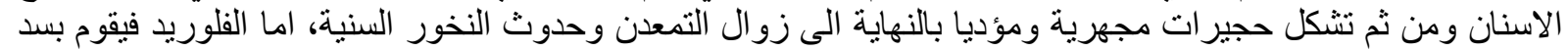

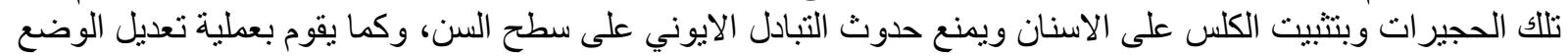

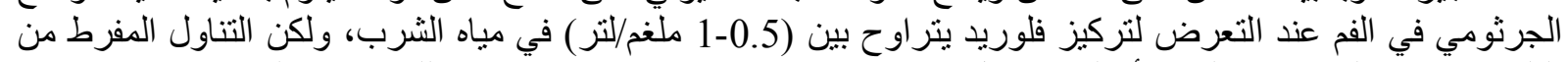

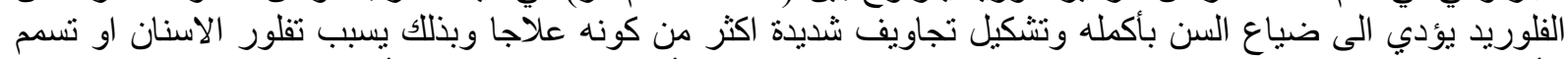

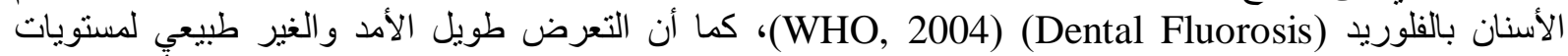

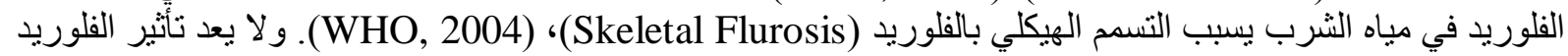

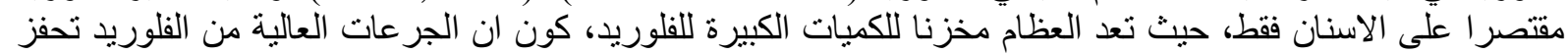

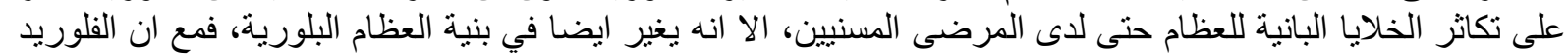

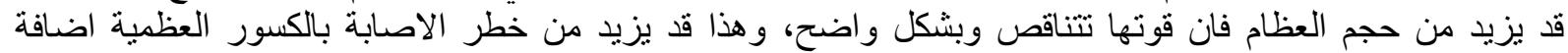

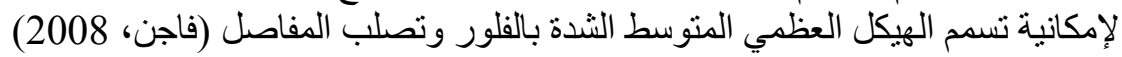

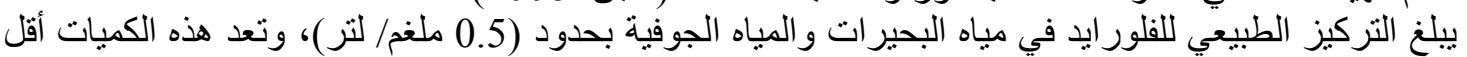

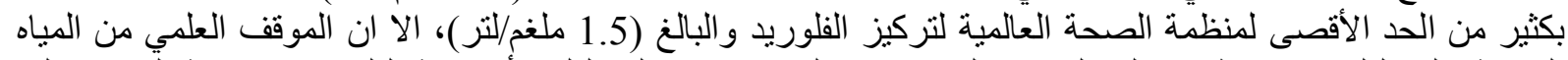

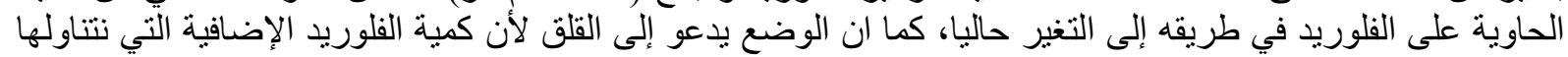

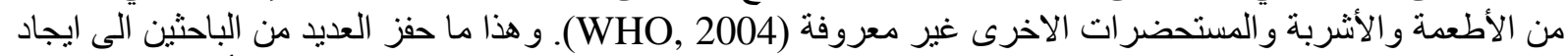

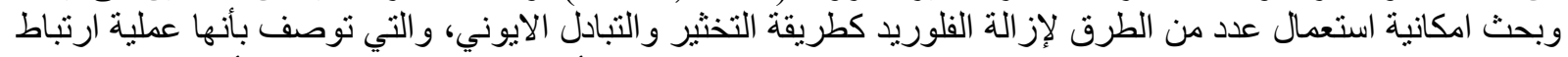

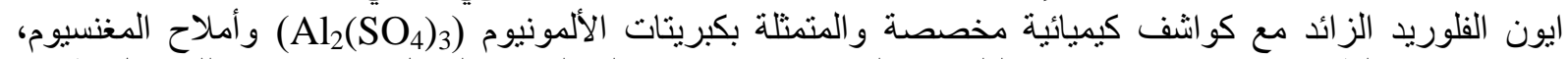

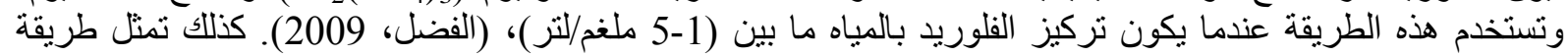

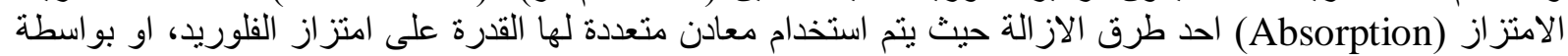

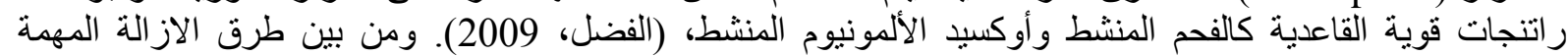

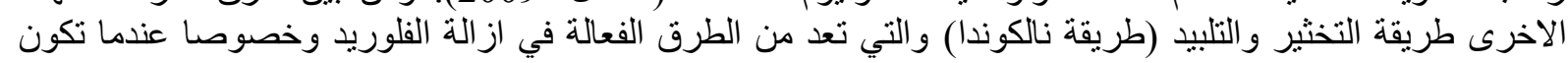

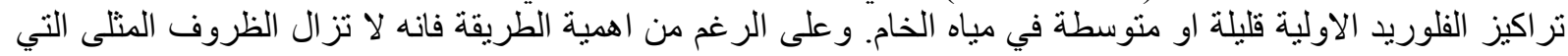

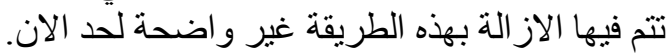

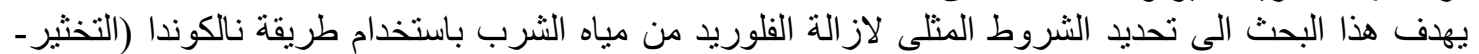

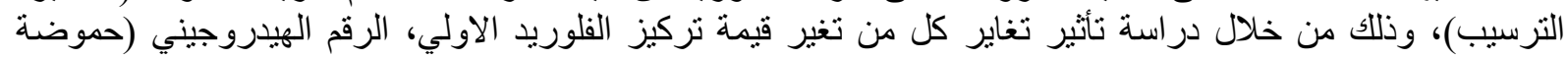

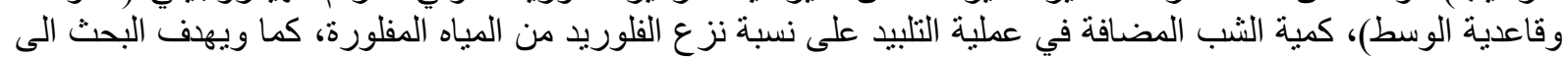

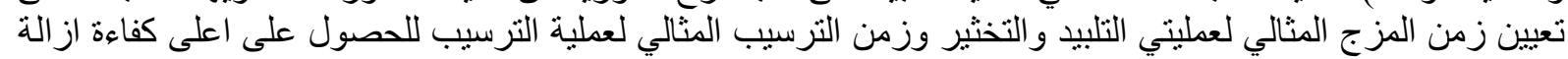
للفلوريد.

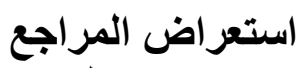

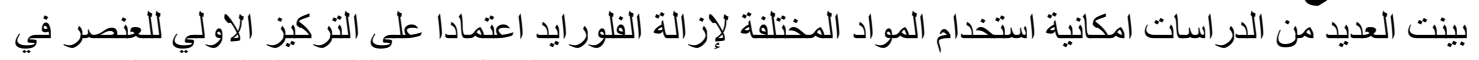

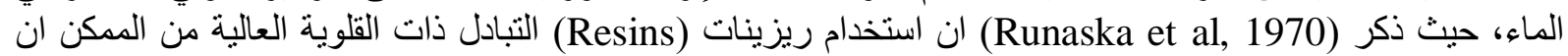

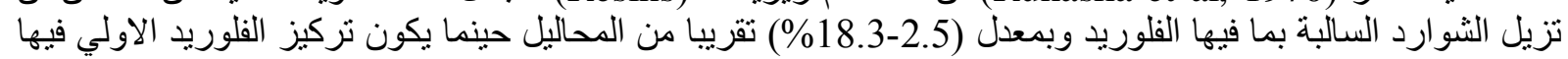

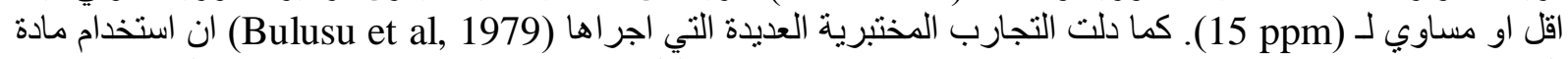

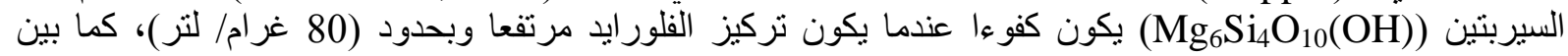

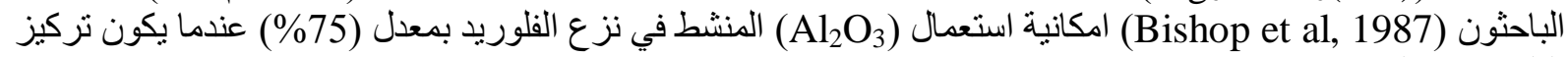
الفلوريد الاولي بحدود (5 ppm). كذلك درس الباحثون (Nawlakhe et al, 1996) فعالية نزع الفلوريد بطريقة نالكوندا عند اضافة المخثر

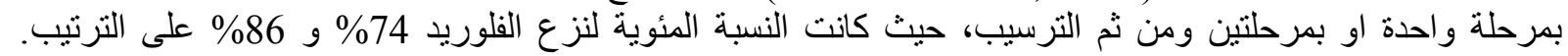

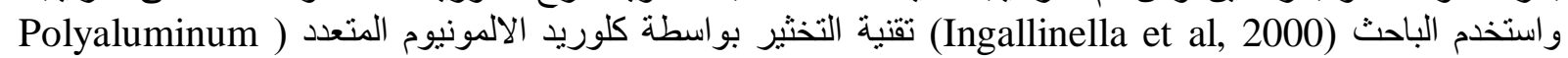


(chloride تليها عملية الترشيح بواسطة مرشح ثنائي الوسط (Dual media filtration) في ازالة الفلورين والزرنيخ

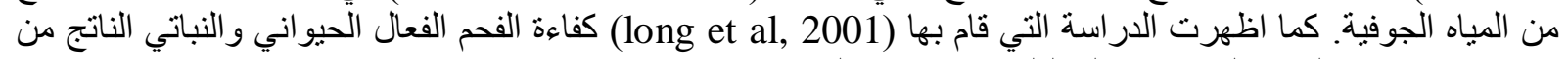

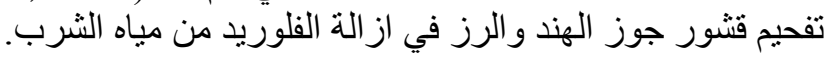

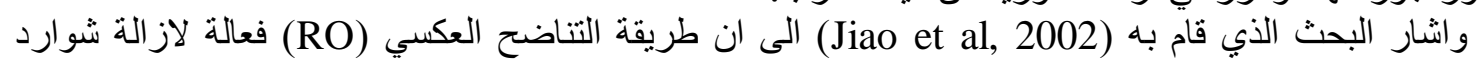

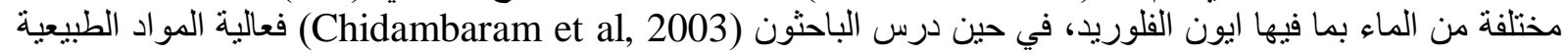

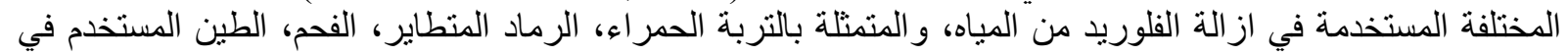

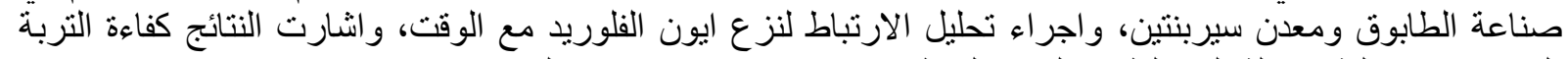

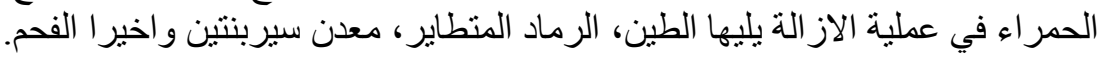

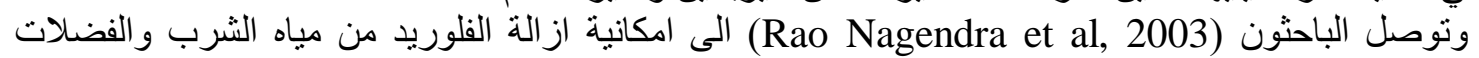

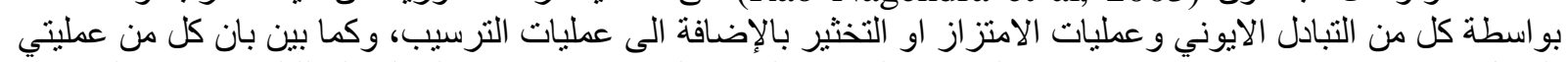

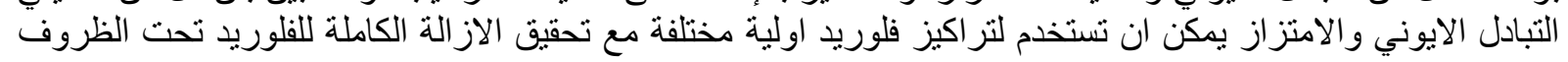
المناسبة الا انها مرتفعة الكلفة.

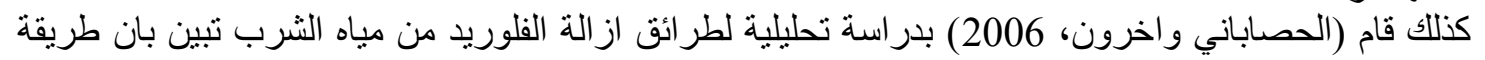

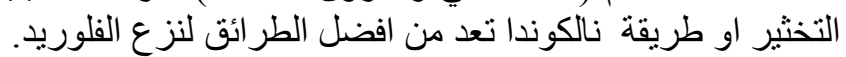

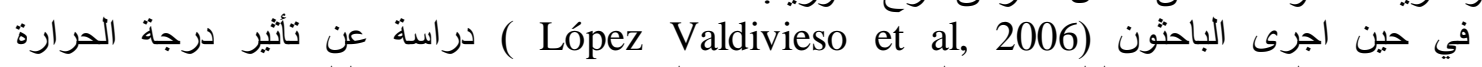

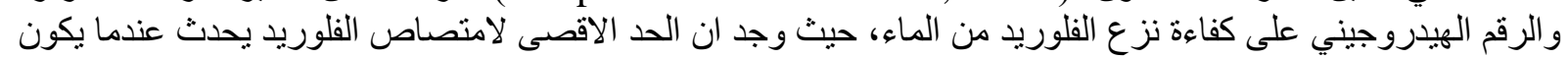

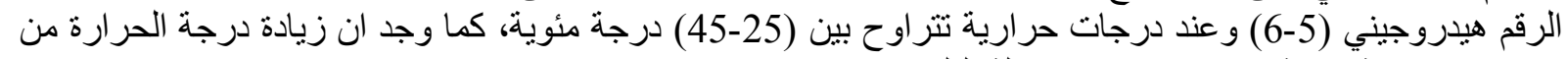
(40-25) درجة مئوية يخفض من كفاءة از الة الفلوريد.

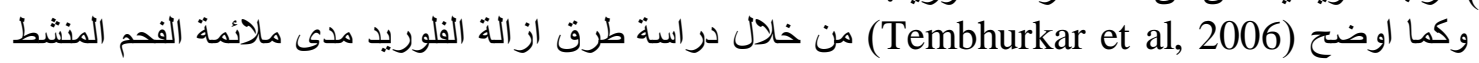

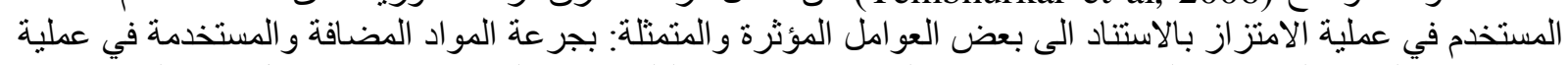

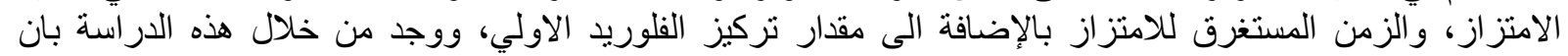

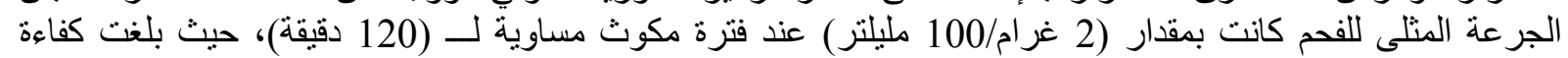
الاز الة عند الظروف المثلى السابقة (94\%).

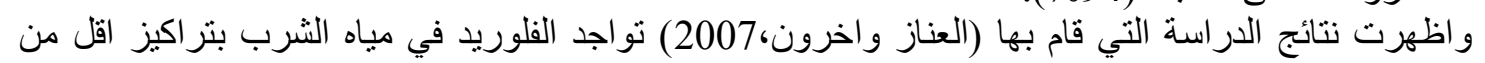

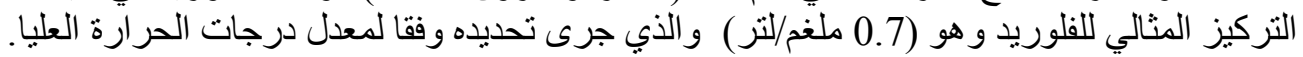

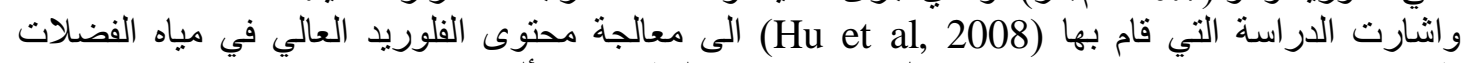

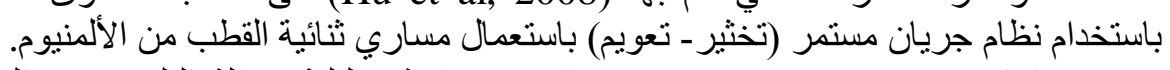

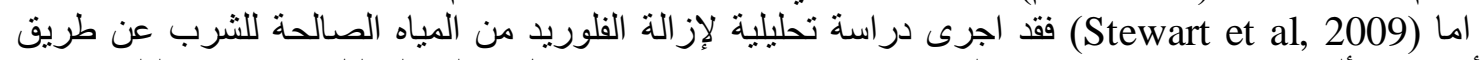

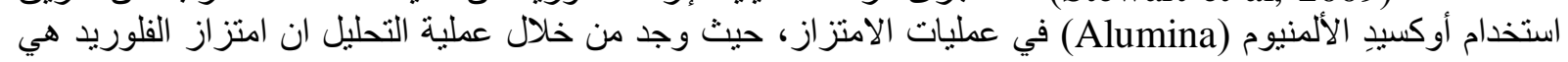

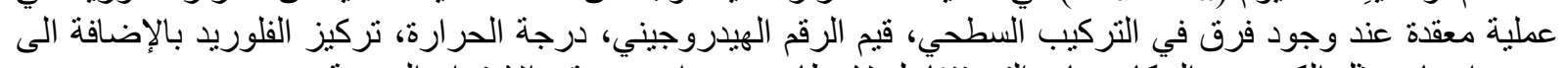

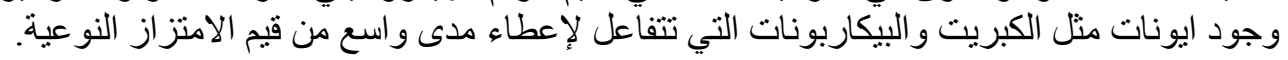

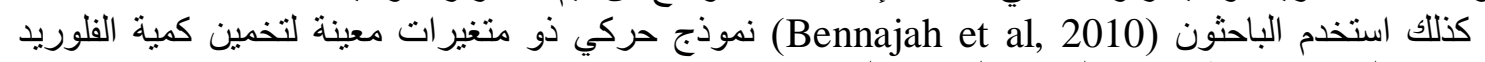
المز الة من مياه الثرب بواسطة تقنية (التخثير -التعويم) الكهربائي.

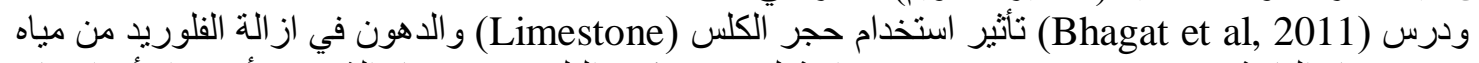

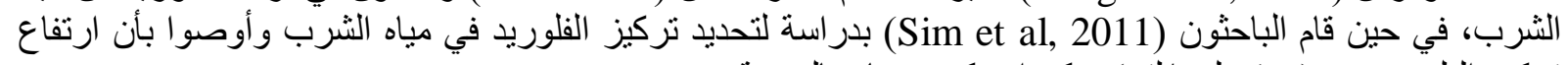
تركيز الفلوريد عن (1.5 ملغم /لتزر ) يمكن ان يكون ضار بالصحة.

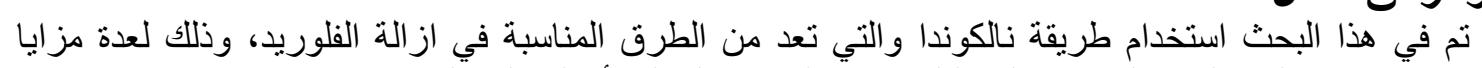

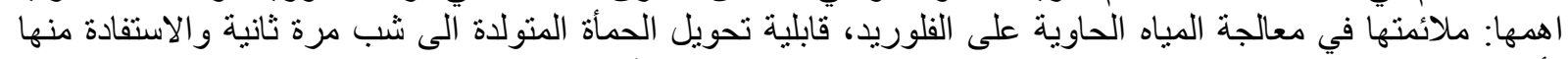

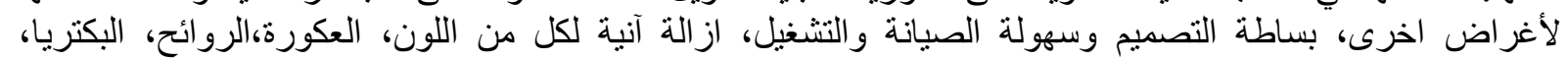

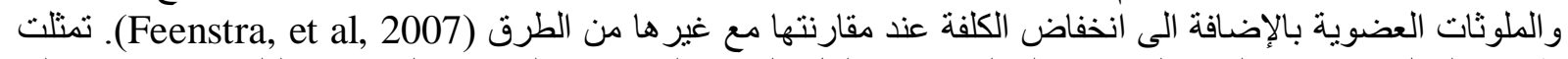

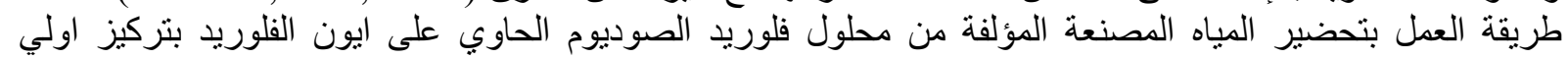

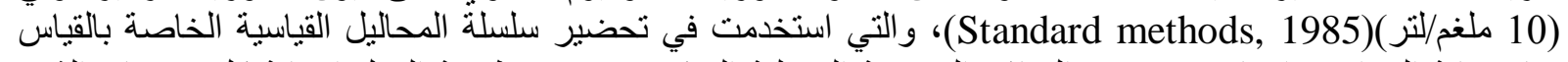

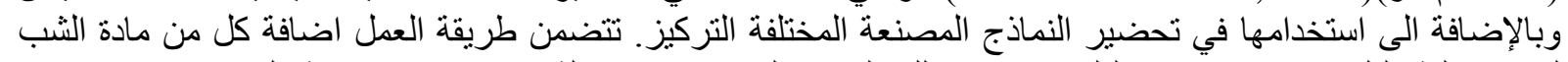

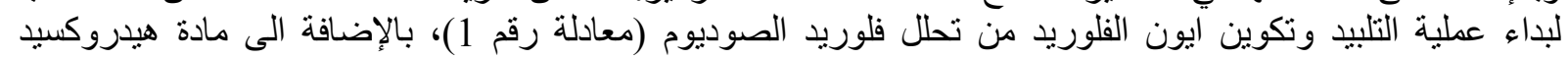

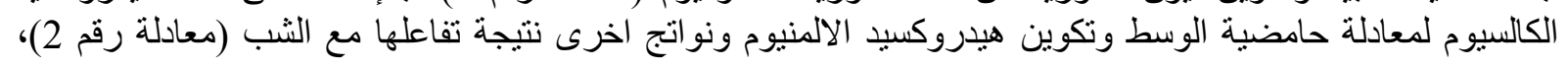




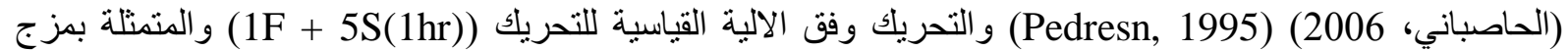

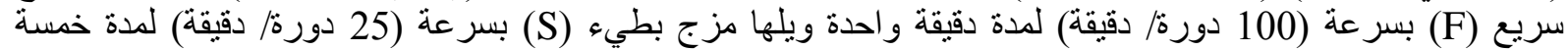

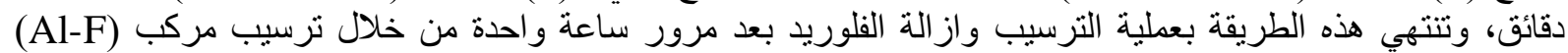

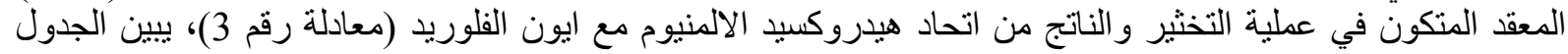

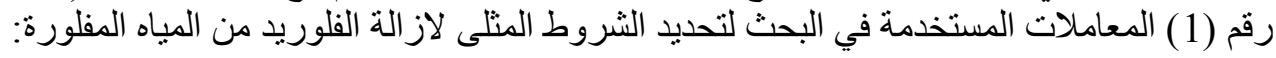

$\mathrm{NaF}+\mathrm{H}_{2} \mathrm{O} \rightarrow \mathrm{F}^{-}+$Other product

$2 \mathrm{KAl}\left(\mathrm{SO}_{4}\right)_{2} \cdot 12 \mathrm{H}_{2} \mathrm{O}+3 \mathrm{Ca}(\mathrm{OH})_{2} \rightarrow 2 \mathrm{Al}(\mathrm{OH})_{3}+3 \mathrm{CaSO}_{4}+\mathrm{K}_{2} \mathrm{SO}_{4}+24 \mathrm{H}_{2} \mathrm{O}$

$\mathrm{Al}(\mathrm{OH})_{3}+\mathrm{F}^{-} \rightarrow(\mathrm{Al}-\mathrm{F})$ complex + Undefined product

الجدول رقم (1) : المر احل و المتغيرات التشغيلية المستخدمة خلال البحث

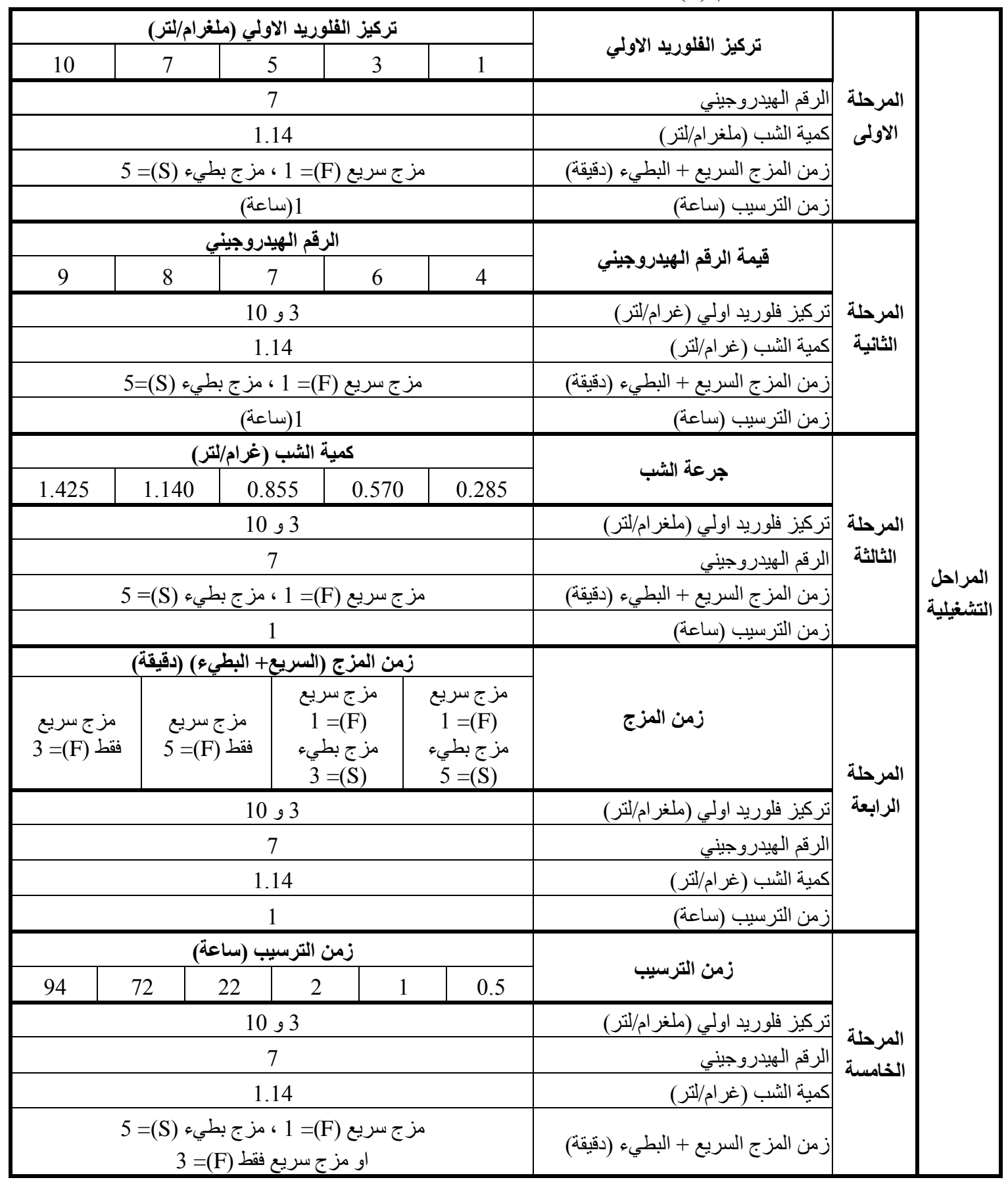




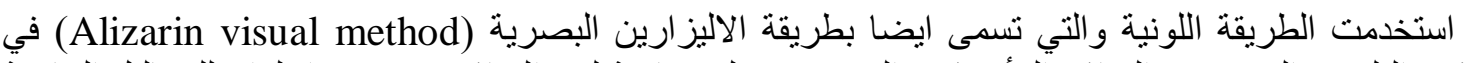

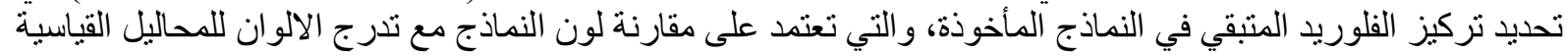
المعلومة التركيز.

\section{النتائج والمناقشتة}

1- تأثير تغير قيمة تركيز الفلوريد الاولي على نسبة نزع الفئ الفلوريد من المياه المفلورة:

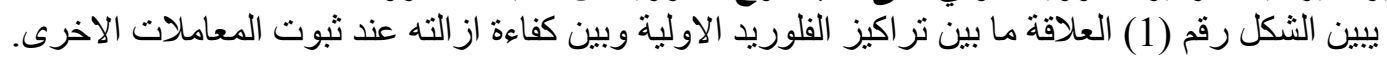

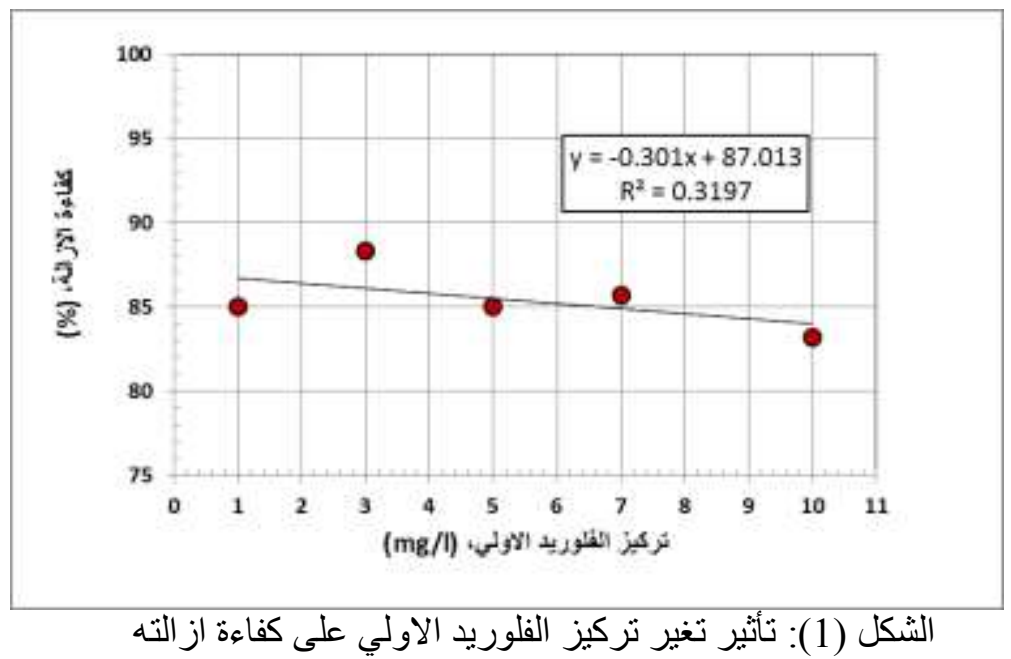

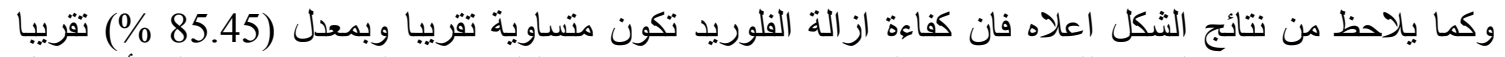

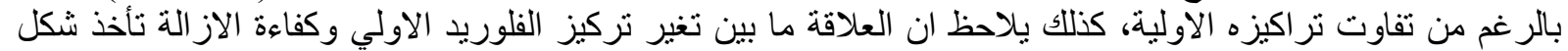

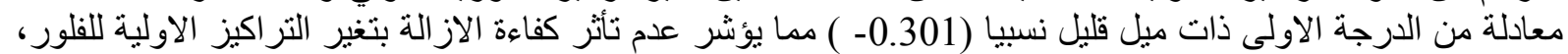

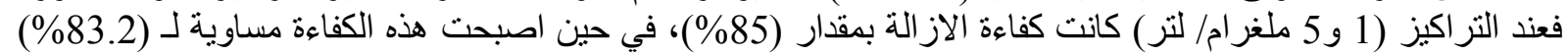

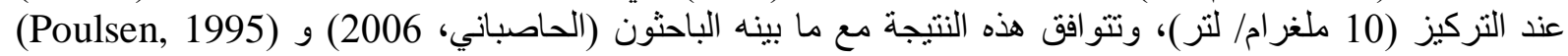

و (Pedersen, 1995) و

2- ت تأثير قيمة الرقم الهيلروجيني (حموضية وقاعدية الوسط) على كفاعة ازالة القلوريد:

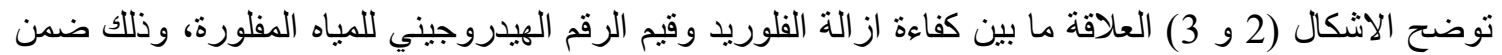

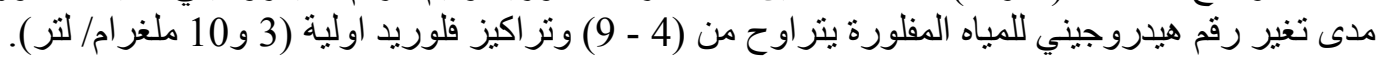

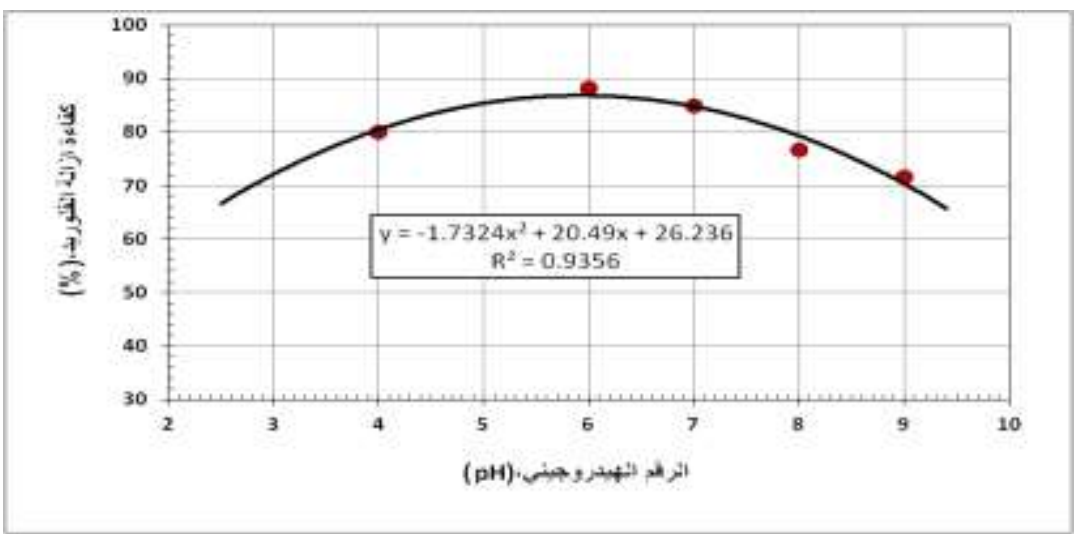

الثكل (2): تأثير الرقم الهيدروجيني على كفاءة ازالة الفلوريد عند تركيز فلوريد اولي (3 ملغرام/ لتر). 


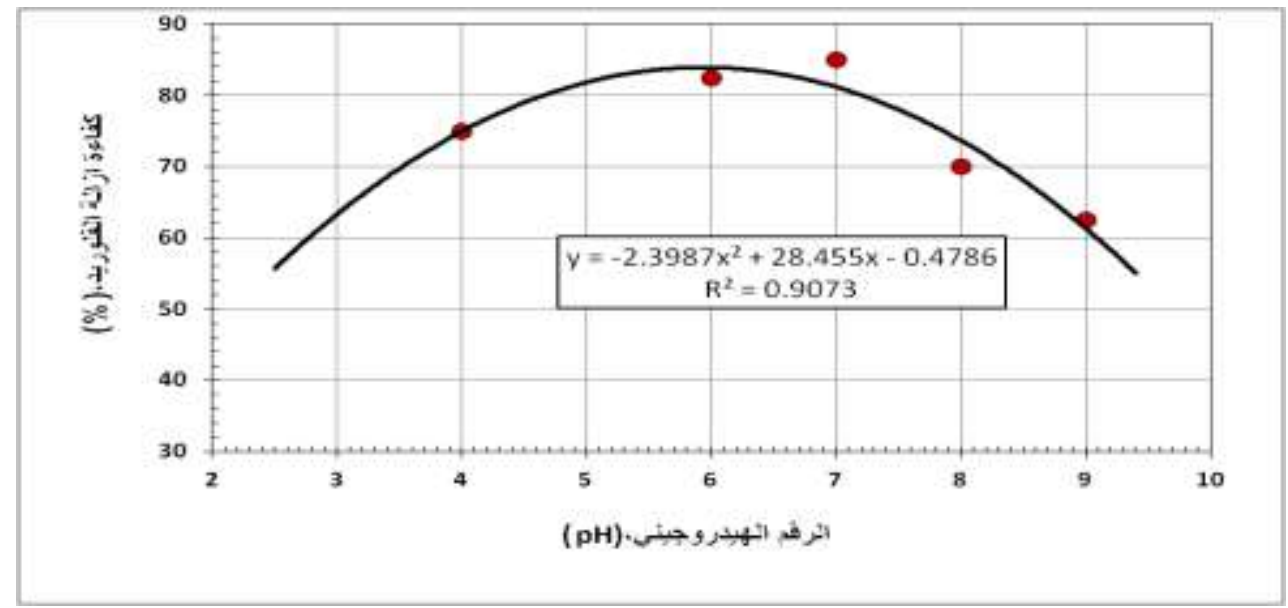

الثكل (3): تأثثر الرقم الهيدروجيني على كفاءة ازالة الفلوريد عند نركيز فلوريد اولي (10 ملغرام/ لتز ).

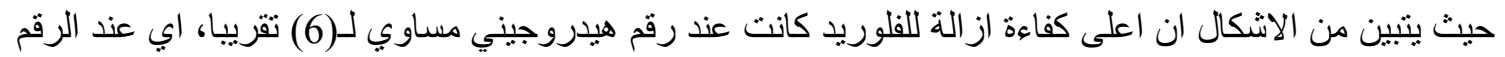

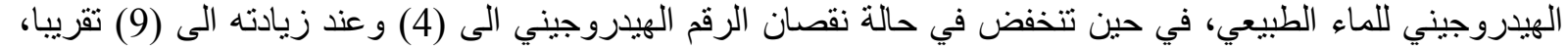

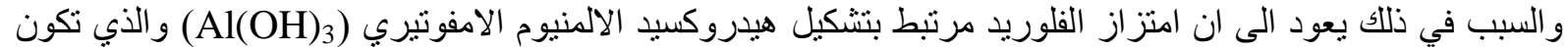

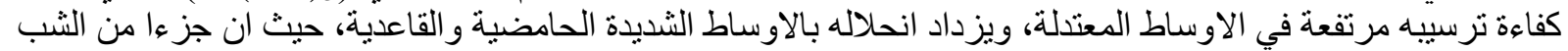

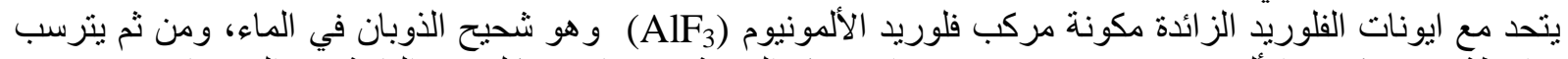

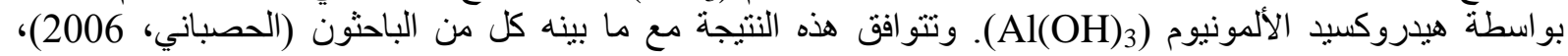
(Azbar, 2000) و (Neeri, 2000)

\section{3- تأثير تغاير كمية الثب المضافة في عملية التلبيد على نسبة نزع الفلوريد:}

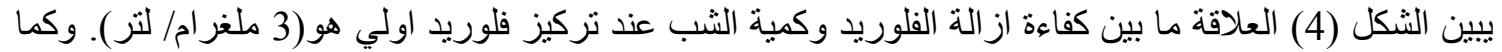

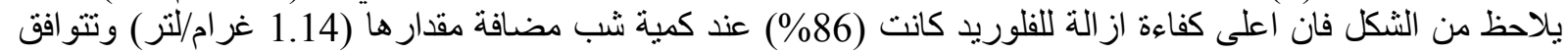

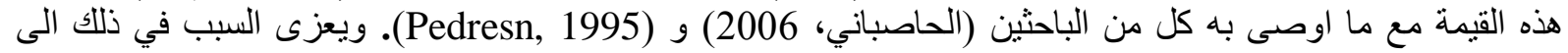

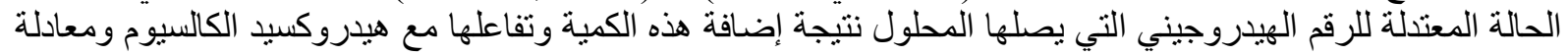

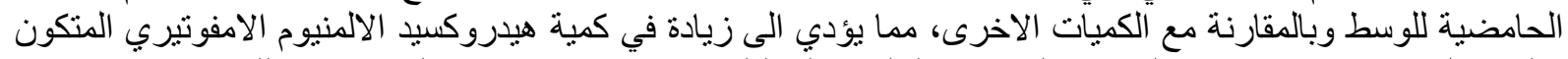

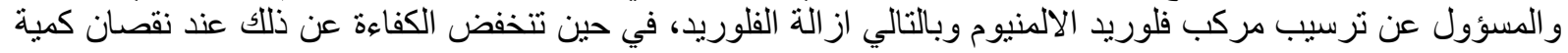

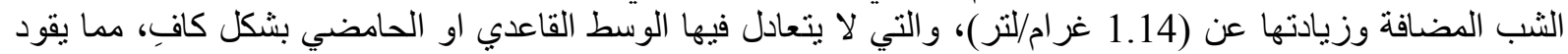

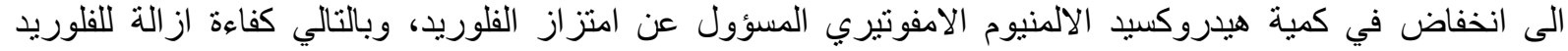
منخفضة، وتتو افق هذه النتائج مع ما بينه كل من (Azbar, 2000)، (Neeri, 2000) و (الحصباني، 2006).

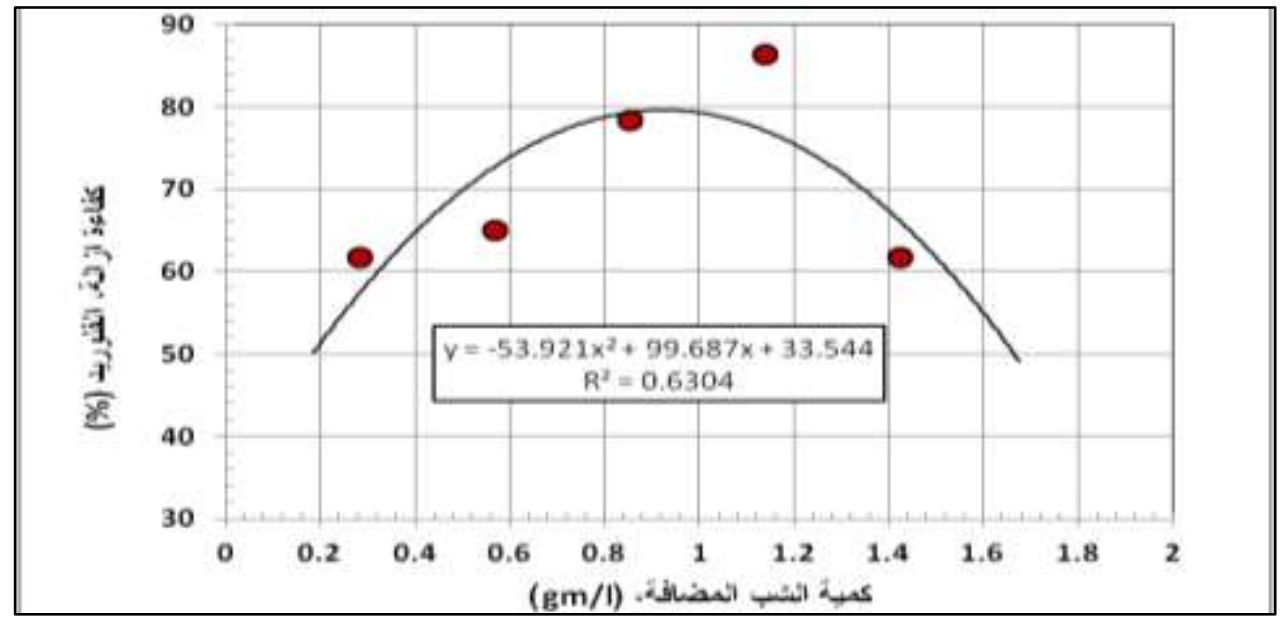

الثكل (4): تغير كمية الثب المضافة على كفاءة ازالة الفلوريد عند تركيز فلوريد اولي (3 ملغرام/ لتر ). 
4- تحديد الزمن المثالي لعمليتي التلبيد و التخثير للحصول على اعلى كفاعة ازالة للفلوريد:

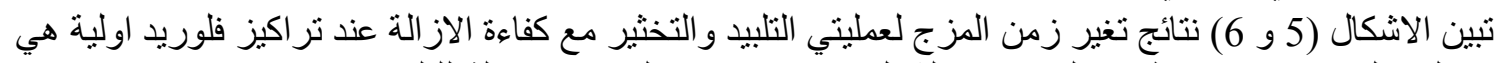

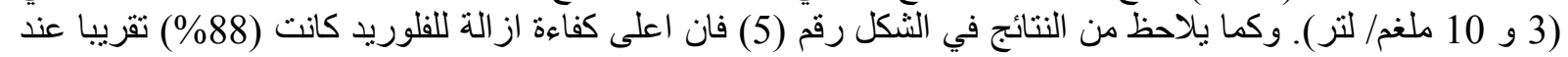

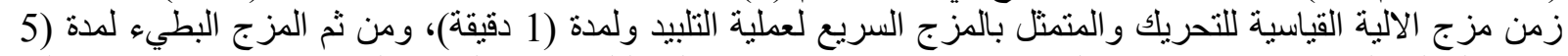

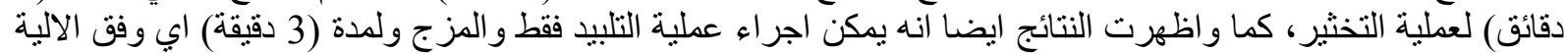

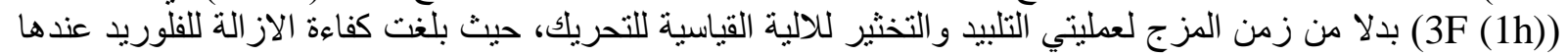
تقريبا (187\%).

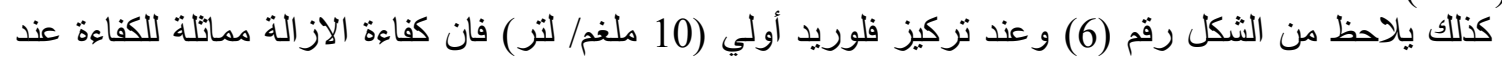

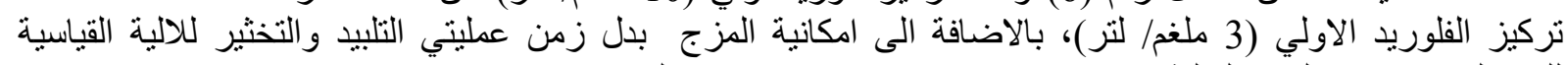

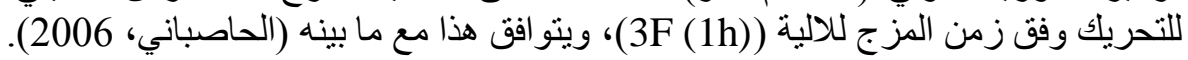

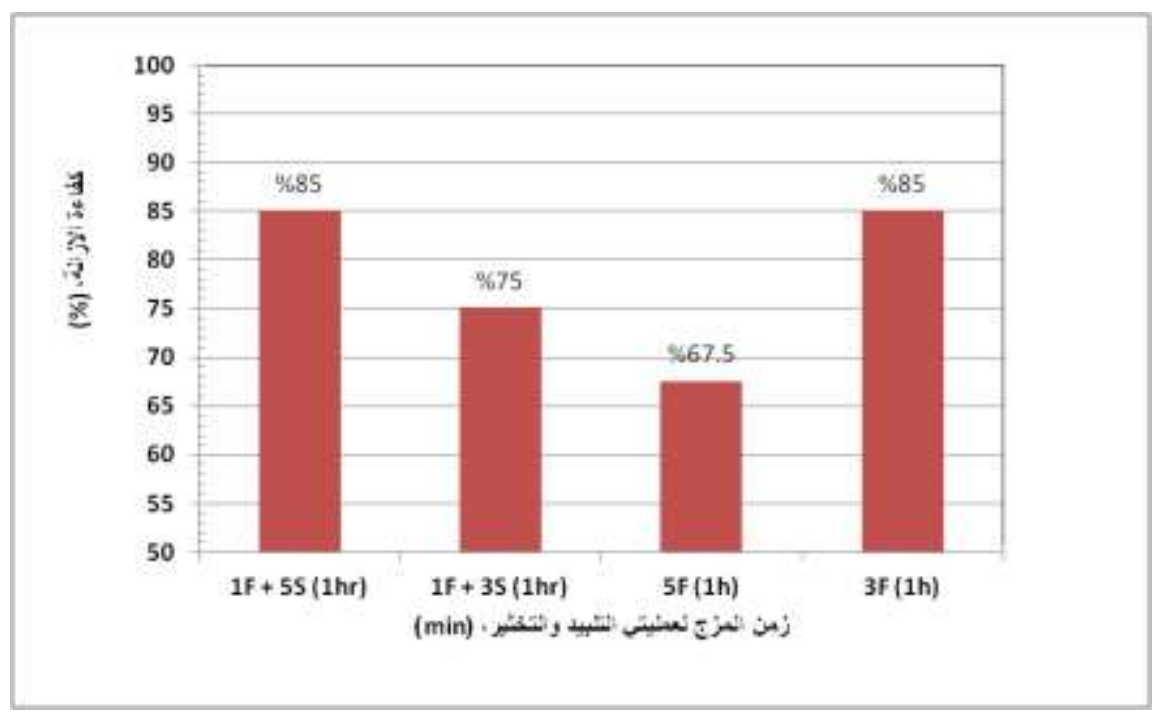

الثكل رقم (5): العلاقة بين كفاءة ازالة الفلوريد وزمن المزج لعمليتي التلبيد والتخثير عند تركيز فلوريد اولي (3 ملغم/ لتز) .

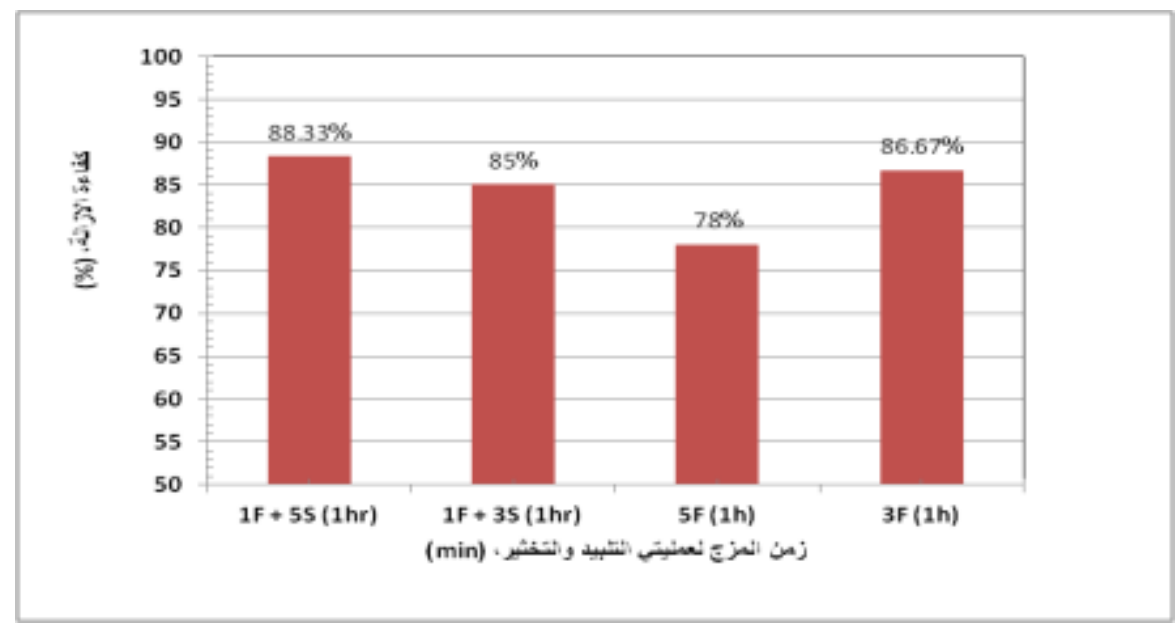

الثكل رقم (6): العلاقة بين كفاءة ازالة الفلوريد وزمن المزج لعمليتي التلبيد والتختير عند تركيز فلوريد اولي (10 ملغة الفوريد لتز ). 
5- تعيين زمن الترسيب المثالي لعملية الترسيب لكفاءة مثالية لازالة لفلوريد:

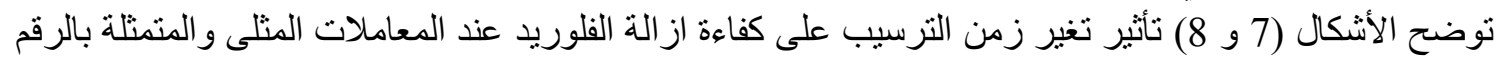

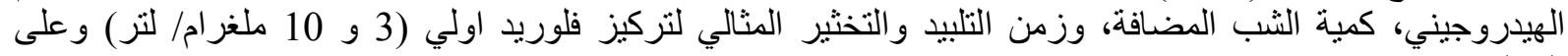
التو الي.

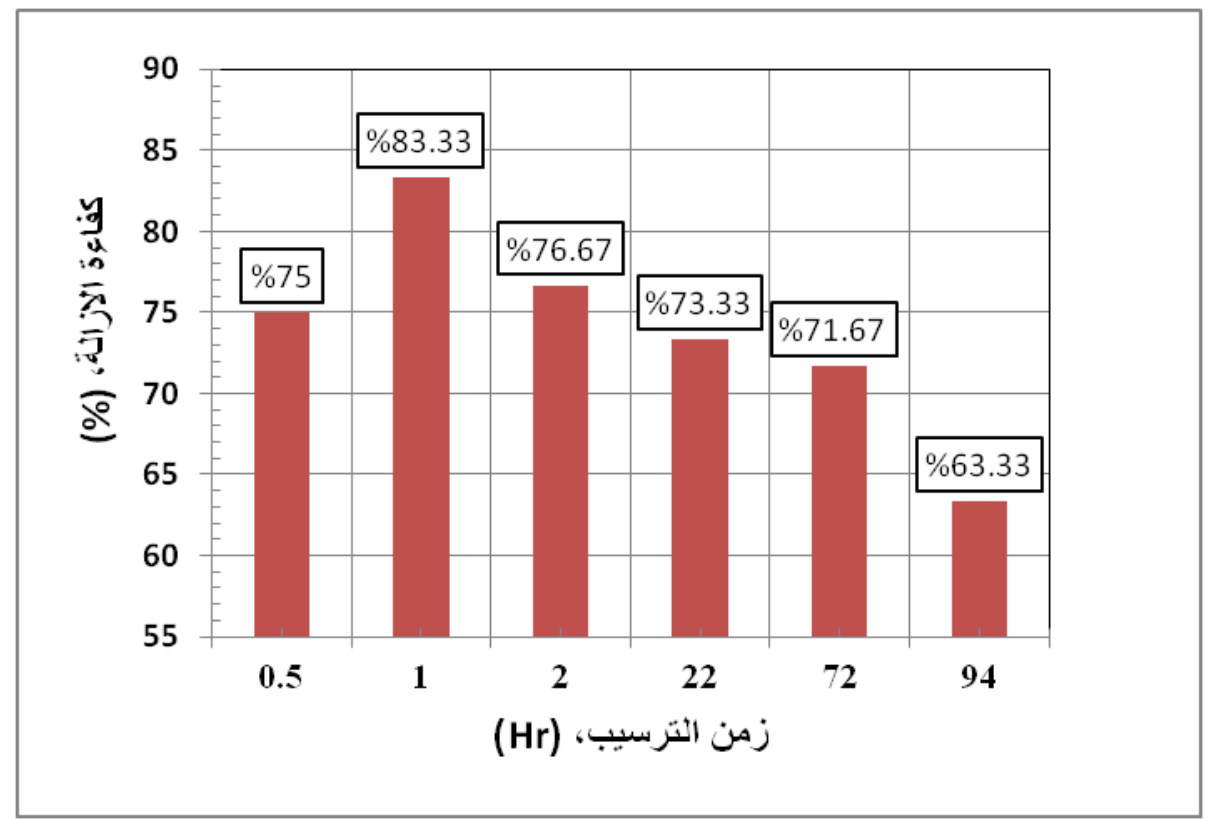

الثكل (7): تأثير زمن الترسيب على كفاءة ازالة الفلوريد عند تركيز فلوريد اولي (3 ملغم/ لنر).

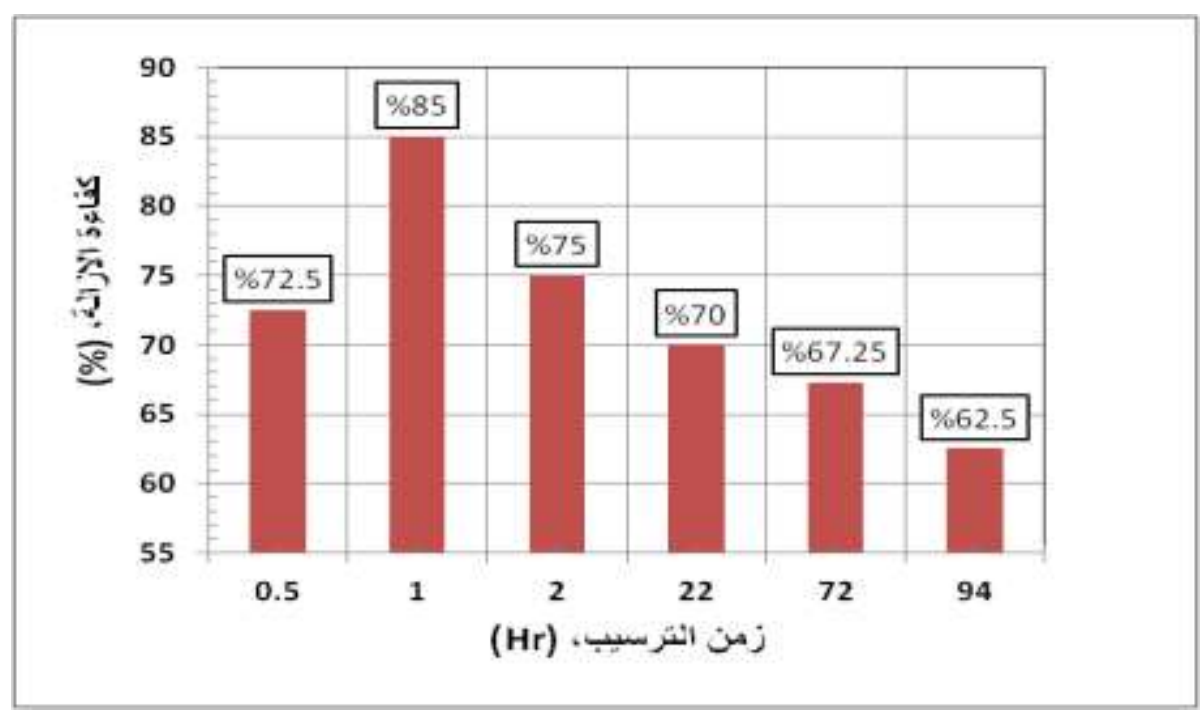

الثكل (8): تأثثر زمن الترسيب على كفاءة ازالة الفلوريد عند تركيز فلوريد اولي (10 ملغخ/ لتز ).

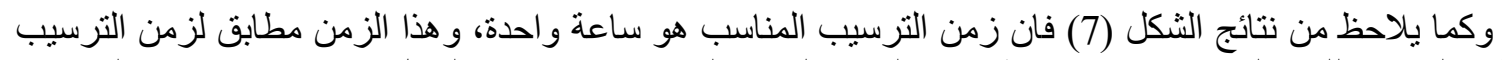

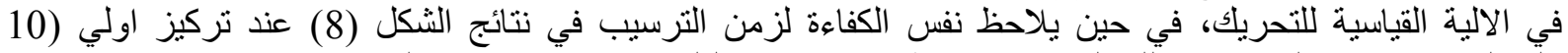
ملغم/لتر)، ويعزى السبب في ذللك الى ان الارنباط ما بين ايون الفلوريد و هيدروكسيد الالمنيوم (Al(

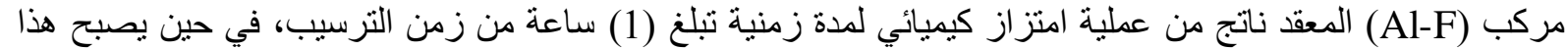

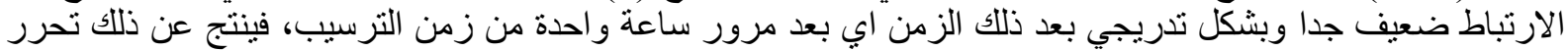

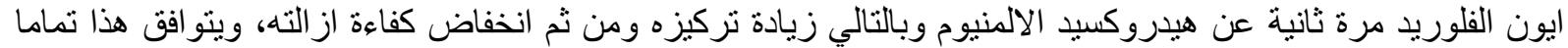

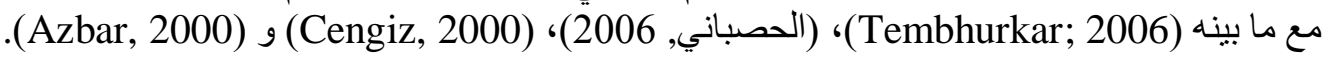


1. وجود تأثير لقيمة التركيز الاولي للفلوريد على كفاءة الاز الة ولكنه قليل، حيث تبقى كفاءة الاز الة نقريبا نفسها عند

تر اكيز فلوريد اولية مختلفة.

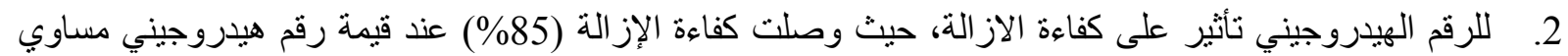

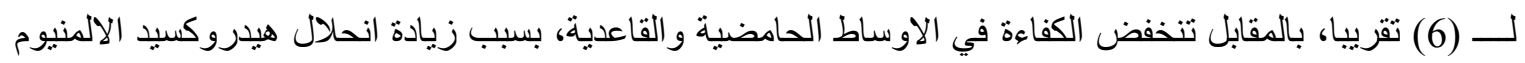

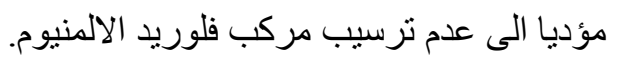

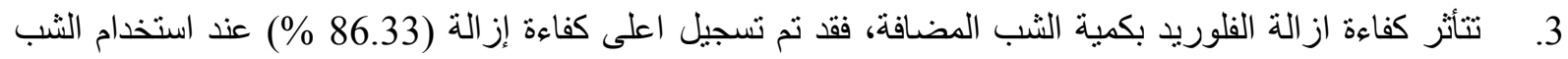

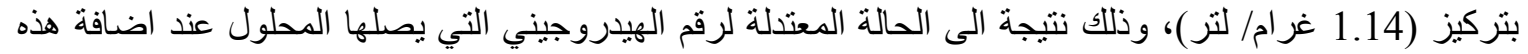
الكمبة من الثب.

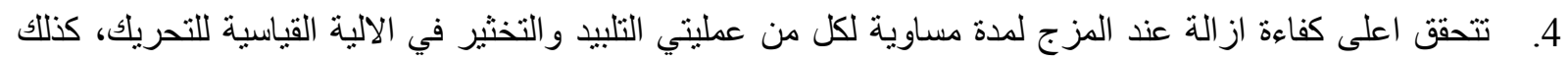

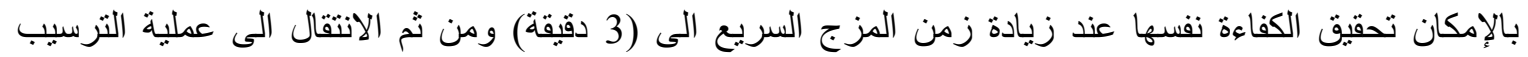

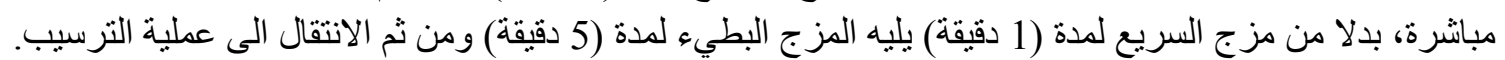

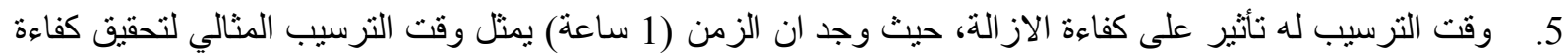

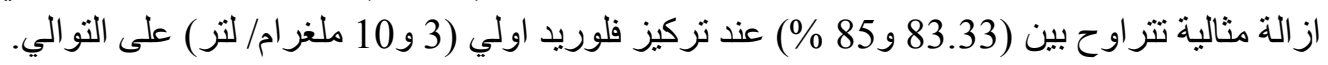

أ- المد المصادر العربية:

1- الحصباني؛ مها ،الجبة؛ ملكة الكية، (1996)، "دراسة طر ائق نزع الفلوريد من مياه الثرب"، (22)، العدد الاول، مجلة جامعة دمثنق للعلوم الاساسية.

2- العناز؛ ليث عبد العليم، عباوي؛ سعاد عبد، (2007)، "تقيبم تراكبز الفلور في مياه الشرب لمدينة الموصل"، مجلة

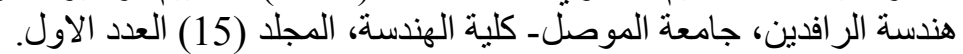

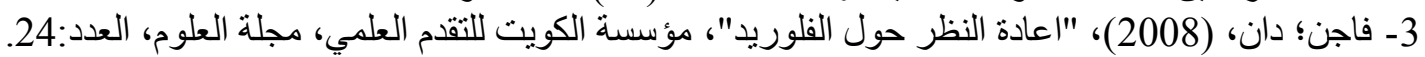

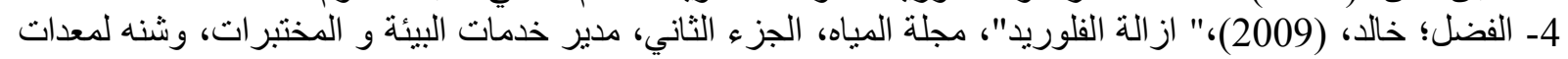
المياه (www.almyah.net).

ب- المصادر الاجنبية:

5. APHA, AWWA and WPCF, (1985), "standard methods for the examination of water and wastewater", American public health association, Washington, D. C., $16^{\text {th }}$ ed.

6. Azbar N. and Turkman A., (2000), "Defluoridation in drinking waters", water science and technology, No.(42), pp. 403-407.

7. Bhagat S. L.; (2011), "Fluoride Removal From Water Using Edible Oil And Crushed Limestone" Science Reviews \& Chemical Communications, vol. (1), No. (1), pp. 49-62.

8. Bennajah M., Darmane Y., Touhami M. E. and Maalmi M., (2010), “A variable order kinetic model to predict defluoridation of drinking water by electrocoagulation electroflotation", International Journal of Engineering, Science and Technology, Vol. (2), No. (12), 2010, pp. 42-52.

9. Bishop, P. L. and Sansoucy, G., (1987), “AWWA.”, (70), pp. 554.

10. Bulusu K. R., Sundaresan B. B., Pathak B. N., Nawlakhe W. G., Kulkarni D. N. and Thergaonkar V. P., (1979), "Fluorides in water, Defluoridation Methods and their Limitations", Vol. (60), pp. 1-25.

11. Cengiz M., Kir E., Kir I. and Fen S. D. ü. (1998), "Bilimleri Enstitü Dergisi", No.(3), p. 69.

12. Chidambaram S., Ramanathan A. L., Vasudevan S., (2003), " Fluoride removal studies in water using natural materials", Water SA, Vol. (29), No. (3) , pp. 339-344.

13. Feenstra 1. ,vasak 1., and Griffioen J., (2007), "fluoride in ground water: over view and evaluation of removal methods". 
14. Gupta S. K. and Deshpande R. D., (1998), "Depleting Groundwater levels and Increasingfluoride concentration in villages of Mehsana District, Gujarat, India: cost to economy and Health", Project Report.

15. Hu C.Y., Lo S. L., Kuan W. H. and Lee Y. D. , (2008), "Treatment of high fluoridecontent wastewater by continuous electrocoagulation - flotation system with bipolar aluminum electrodes" Separation and Purification Technology, vol. (60), pp.1-5.

16. Ingallinella A. M., Fernández R. G. and Stecca L. M., (2000), "Arsenic and fluorine removal from ground water by coagulation with polyaluminum chloride and double filtration" cis@fceia.unr.edu.ar.

17. Jiao Z., Zhang Y., Yang M. and Huaang X. M., (2002), "Removal Fluoride using rare earth based inorganic adsorbent", vol. (21), No. (4), pp. 365-370.

18. Long R. Q. and Yang R. T., (2001), "Carbon Nanotubes as Superior Sorbent for Dioxin Removal", American Chemical Society, No. (123), pp. 2058.

19. López Valdivieso A, Reyes Bahena J. L., Song S, and Herrera Urbina R., (2006), " Temperature effect on the zeta potential and fluoride adsorption at the alphaAl2O3/aqueous solution interface", J. Colloid Interface Sci., vol. (298), No. (1), pp.1-5.

20. Müller K., Johnson A., Meierhofer R. and Wegelin M., (2006), "Fluoride Removal in Developing Countries: State-of-the Art of Defluoridation Techniques in East Africa", Sandec News, No. (7), pp. 12-13.

21. Nawlakhe W. G., Bulusu K. R., Ppathak B. N. and Paramasivam R., (1996), "Settling Characteristic of Flocculent Suspension in Defluovidation of Water by Nalgonda Technique", Water Chemistry Division, NEERI, Nagpur, India, No. (30), p. 103.

22. Neeri D., (2000), "Defluoridation technology mission on drinking water in villages and related water management", National Environment Engineering Research institute Nagpur India, No.(77), p. 20.

23. Pedersen A., (1995), "Optimization and pilot implementation of the Nalgonda technique on household level for use in Tanzania", Technical university of Denmark, No.(18), pp. 21-33.

24. Poulsen B. K. (1995), "Optimization of the Nalgonda Technique Adding Different Ghemicals", Technical. University of Denmark, No.(41), p. 34.

25. Rao Nagendra C.R., (2003) "Fluoride And Environment- A Review", Proceedings of the Third International Conference on Environment and Health, University of Madras and Faculty of Environmental Studies, York University, pp. 386 - 399.

26. Runaska W., Kawane M. and Kajima T., (1970), "Removal of Fluorides Ion by Anion Exchange Resin", Chemical Abstracts, No.(45), p. 5033.

27. Sim J. M. and Leong K. M., (2011), "Feasibility study on fluoride removal in drinking water in Mehsana, India", International NGO Journal, Vol. (6), No. (10), pp. 224-228.

28. Stewart T., (2009), "Removal of Fluoride from Drinking Water: Analysis of Alumina Based Sorption", Institute of Biogeochemistry and Pollutant Dynamics, Department of Environmental Sciences.

29. Tembhurkar A. R. and Dongre S., (2006), "studies on fluoride removal using adsorption process", Journal of Environ. science \& Eng., vol. (48), No. (3), pp.151-156.

30. World Health Organisation, (2004), "Guideline for drinking water supply", Geneva, Switzerland, 3rd Edition.

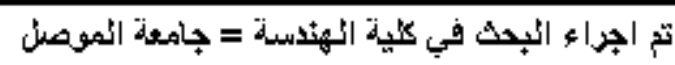

